\title{
Crossing and Weighted Crossing Number of Near-Planar Graphs
}

\author{
Sergio Cabello ${ }^{1, \star}$ and Bojan Mohar ${ }^{2, \star \star, \star \star \star}$ \\ ${ }^{1}$ Department of Mathematics, FMF, University of Ljubljana \\ sergio.cabello@fmf.uni-lj.si \\ ${ }^{2}$ Department of Mathematics, Simon Fraser University, Burnaby, B.C. V5A 1S6 \\ mohar@sfu.ca
}

\begin{abstract}
A nonplanar graph $G$ is near-planar if it contains an edge $e$ such that $G-e$ is planar. The problem of determining the crossing number of a near-planar graph is exhibited from different combinatorial viewpoints. On the one hand, we develop min-max formulas involving efficiently computable lower and upper bounds. These min-max results are the first of their kind in the study of crossing numbers and improve the approximation factor for the approximation algorithm given by Hliněný and Salazar (Graph Drawing GD 2006). On the other hand, we show that it is NP-hard to compute a weighted version of the crossing number for near-planar graphs.
\end{abstract}

\section{Introduction}

Crossing number minimization is one of the fundamental optimization problems in the sense that it is related to various other widely used notions. Besides its mathematical interest, there are numerous applications, most notably those in VLSI design [1819, in combinatorial geometry and even in number theory, see, e.g, [16. We refer to [10]15] and to [18 for more details about diverse applications of this important notion.

A nonplanar graph $G$ is near-planar if it contains an edge $e$ such that $G-e$ is planar. Such an edge $e$ is called a planarizing edge. It is easy to see that nearplanar graphs can have arbitrarily large crossing number. However, it seems that computing the crossing number of near-planar graphs should be much easier than in unrestricted cases. This is supported by a less known, but particularly interesting result of Riskin [14, who proved that the crossing number of a 3connected cubic near-planar graph $G$ can be computed easily as the length of a shortest path in the geometric dual graph of the planar subgraph $G-x-y$, where

* Supported in part by the Slovenian Research Agency, project J1-7218 and program P1-0297.

${ }^{\star *}$ Supported in part by the ARRS, Research Program P1-0297, by an NSERC Discovery Grant, and by the Canada Research Chair Program.

$\star \star \star$ On leave from IMFM \& FMF, Department of Mathematics, University of Ljubljana, 1000 Ljubljana, Slovenia. 
$x y \in E(G)$ is the edge whose removal yields a planar graph. Riskin asked if a similar correspondence holds in more general situations, but this was disproved by Mohar [13. (see also [5]). Another relevant paper about crossing numbers of near-planar graphs was published by Hliněný and Salazar [6].

In this paper we show that several generalizations of Riskin's result are indeed possible. We provide efficiently computable upper and lower bounds on the crossing number of near-planar graphs in a form of min-max relations. These relations can be extended to the non-3-connected case and even to the case of weighted edges. As far as we know, these results are the first of their kind in the study of crossing numbers. It is shown that they generalize and improve some known results and we foresee that generalizations and further applications are possible.

On the other hand, we show that computing the crossing number of weighted near-planar graphs is NP-hard. This discovery is a surprise and brings more questions than answers.

Drawings and crossings. A drawing of a graph $G$ is a representation of $G$ in the Euclidean plane $\mathbb{R}^{2}$ where vertices are represented as distinct points and edges by simple polygonal arcs joining points that correspond to their endvertices. A drawing is clean if the interior of every arc representing an edge contains no points representing the vertices of $G$. If interiors of two arcs intersect or if an arc contains a vertex of $G$ in its interior we speak about crossings of the drawing. More precisely, a crossing of a drawing $\mathcal{D}$ is a pair $(\{e, f\}, p)$, where $e$ and $f$ are distinct edges and $p \in \mathbb{R}^{2}$ is a point that belongs to interiors of both arcs representing $e$ and $f$ in $\mathcal{D}$. If the drawing is not clean, then the arc of an edge $e$ may contain in its interior a point $p \in \mathbb{R}^{2}$ that represents a vertex $v$ of $G$. In such a case, the pair $(\{v, e\}, p)$ is also referred to as a crossing of $\mathcal{D}$.

The number of crossings of $\mathcal{D}$ is denoted by $\operatorname{cr}(\mathcal{D})$ and is called the crossing number of the drawing $\mathcal{D}$. The crossing number $\operatorname{cr}(G)$ of a graph $G$ is the minimum $\operatorname{cr}(\mathcal{D})$ taken over all clean drawings $\mathcal{D}$ of $G$. When each edge $e$ of $G$ has a weight $w_{e} \in \mathbb{N}$, the weighted crossing number $\operatorname{wcr}(\mathcal{D})$ of a clean drawing $\mathcal{D}$ is the sum $\sum w_{e} \cdot w_{f}$ over all crossings $(\{e, f\}, p)$ in $\mathcal{D}$. The weighted crossing number $\operatorname{wcr}(G)$ of $G$ is the minimum wcr $(\mathcal{D})$ taken over all clean drawings $\mathcal{D}$ of $G$. Of course, if all edge-weights are equal to 1 , then $\operatorname{wcr}(G)=\operatorname{cr}(G)$.

We shall discuss both, the weighted and unweighted crossing number. Most of the results are treated for the general weighted case. However, some results hold only in the unweighted case or are too technical to state for the weighted case. For a graph we shall assume that it is unweighted (i.e., all edge-weights are equal to 1) unless stated explicitly or when it is clear from the context that it is weighted.

A clean drawing $\mathcal{D}$ with $\operatorname{cr}(\mathcal{D})=0$ is also called an embedding of $G$. By a plane graph we refer to a planar graph together with a fixed embedding in the Euclidean plane. We shall identify a plane graph with its image in the plane.

Dual and facial distances. Let $G_{0}$ be a plane graph and let $x, y$ be two of its vertices. A simple (polygonal) arc $\gamma:[0,1] \rightarrow \mathbb{R}^{2}$ is an $(x, y)$-arc if $\gamma(0)=x$ and 
$\gamma(1)=y$. If $\gamma(t)$ is not a vertex of $G_{0}$ for every $t, 0<t<1$, then we say that $\gamma$ is clean. For an $(x, y)$-arc $\gamma$ we define the crossing number of $\gamma$ with $G_{0}$ as

$$
\operatorname{cr}\left(\gamma, G_{0}\right)=\mid\left\{t \mid \gamma(t) \in G_{0} \text { and } 0<t<1\right\} \mid .
$$

This definition extends to the weighted case as follows. If the graph $G_{0}$ is weighted and the edge $x y$ realized by an $(x, y)$-arc $\gamma$ also has weight $w_{x y}$, then each crossing of $\gamma$ with an edge $e$ contributes $w_{x y} \cdot w_{e}$ towards the value $\operatorname{cr}\left(\gamma, G_{0}\right)$, and each crossing $(\{v, x y\}, p)$ of $x y$ with a vertex of $G_{0}$ contributes 1 (independently of the edge-weights).

Using this notation, we define the dual distance

$$
d^{*}(x, y)=\min \left\{\operatorname{cr}\left(\gamma, G_{0}\right) \mid \gamma \text { is a clean }(x, y)-\operatorname{arc}\right\}
$$

We also introduce a similar quantity, the facial distance between $x$ and $y$ :

$$
d^{\prime}(x, y)=\min \left\{\operatorname{cr}\left(\gamma, G_{0}\right) \mid \gamma \text { is an }(x, y)-\operatorname{arc}\right\}
$$

It should be observed at this point that the value $d^{\prime}(x, y)$ is independent of the weights - since all weights are integers, we can replace each crossing of an edge with a crossing through an incident vertex and henceforth replace weight contributions simply by counting the number of crossings.

Let $G_{x, y}^{*}$ be the geometric dual graph of $G_{0}-x-y$. Then $d^{*}(x, y)$ is equal to the distance in $G_{x, y}^{*}$ between the two vertices corresponding to the faces of $G_{0}-x-y$ containing $x$ and $y$. Of course, in the weighted case the distances are determined by the weights of their dual edges. This shows that $d^{*}(x, y)$ can be computed in linear time by using known shortest path algorithms for planar graphs. Similarly, one can compute $d^{\prime}(x, y)$ in linear time by using the vertex-face incidence graph (see [12]).

Clearly, $d^{\prime}(x, y) \leq d^{*}(x, y)$. Note that $d^{*}$ and $d^{\prime}$ depend on the embedding of $G_{0}$ in the plane. However, if $G_{0}$ is (a subdivision of) a 3-connected graph, then this dependency disappears since $G_{0}$ has essentially a unique embedding. To compensate for this dependence, we define $d_{0}^{*}(x, y)$ (and $d_{0}^{\prime}(x, y)$ ) as the minimum of $d^{*}(x, y)$ (resp. $d^{\prime}(x, y)$ ) taken over all embeddings of $G_{0}$ in the plane.

Overview of results. The following proposition is clear from the definition of $d^{*}$ :

Proposition 1. If $G_{0}$ is a weighted planar graph and $x, y \in V\left(G_{0}\right)$, then $\operatorname{cr}\left(G_{0}+x y\right) \leq d_{0}^{*}(x, y)$.

This result shows that the value $d_{0}^{*}(x, y)$ is of interest. Gutwenger, Mutzel, and Weiskircher [5] provided a linear-time algorithm to compute $d_{0}^{*}(x, y)$. In Sect. 2 we study $d_{0}^{*}(x, y)$ from a combinatorial point of view and obtain a min-max characterization that results very useful.

Riskin 14 proved the following strengthening of Proposition 1 in a special case when $G_{0}$ is 3 -connected and cubic: 
Theorem 1 ([14]). If $G_{0}$ is a 3-connected cubic planar graph, then

$$
\operatorname{cr}\left(G_{0}+x y\right)=d_{0}^{*}(x, y)
$$

Riskin asked in [14] if Theorem[1]extends to arbitrary 3-connected planar graphs. One of the authors 13 has shown that this is not the case: for every integer $k$, there exists a 5-connected planar graph $G_{0}$ and two vertices $x, y \in V\left(G_{0}\right)$ such that $\operatorname{cr}\left(G_{0}+x y\right) \leq 11$ and $d_{0}^{*}(x, y) \geq k$. See also Gutwenger, Mutzel, and Weiskircher [5] for an alternative construction.

However, several extensions of Theorem 1 are possible, and some of them are presented throughout this paper. In particular, we show how to deal with graphs that are not 3-connected, and what happens when we allow vertices of arbitrary degrees.

Theorem 2. If $G_{0}$ is a weighted planar graph and $x, y \in V\left(G_{0}\right)$, then

$$
d_{0}^{\prime}(x, y) \leq \operatorname{cr}\left(G_{0}+x y\right) \leq d_{0}^{*}(x, y) .
$$

The proof of this result is given in Sect. 3 .

If $G_{0}$ is a cubic graph, then for every planar embedding of $G_{0}, d^{\prime}(x, y)=$ $d^{*}(x, y)$. Therefore, $d_{0}^{\prime}(x, y)=d_{0}^{*}(x, y)$, and Theorem 2 implies Theorem 1 .

Theorem 2 is also the main ingredient to improve the approximation factor in the algorithm of Hliněný and Salazar 6; see Corollary 3 .

A key idea in our results is to show that $d_{0}^{*}(x, y)$ (respectively $d_{0}^{\prime}(x, y)$ ) is closely related to the maximum number of edge-disjoint (respectively vertexdisjoint) cycles that separate $x$ and $y$. The notion of the separation has to be understood in a certain strong sense that is introduced in Sect. 2 This result yields a dual expression for $d_{0}^{*}$ (respectively $d_{0}^{\prime}$ ) and is used to show that $d_{0}^{*}(x, y)$ is closely related to the crossing number of $G_{0}+x y$, while $d_{0}^{\prime}(x, y)$ is in the same way related to the minor crossing number, $\operatorname{mcr}\left(G_{0}+x y\right)$, a version of the crossing number that works well with minors; see Bokal et al. [2].

Finally, we show in Sect. 5 that computing the crossing number of weighted near-planar graphs is NP-hard. Our reduction uses weights that are not polynomially bounded, and therefore it does not imply NP-hardness for unweighted graphs.

Intuition. To understand the difficulty in computing the crossing number of a near-planar graph, let us consider the graph shown in Fig. 1 (taken from [13]), where the subgraph inside each of the "darker" triangles is a sufficiently dense triangulation that requires many crossings when crossed by an arc. By drawing the vertex $x$ in the outside, we see that this graph is near-planar. The drawing in Fig. 1 shows that its crossing number is at most 11, but it is also clear that $d^{*}(x, y)$ can be made as large as we want.

This construction can be generalized such that a similar redrawing as made there for $x$ is necessary also for $y$ (in order to bring these two vertices "close together"). At the first sight this seems like the only possibility which may happen - to "flip" a part of the graph containing $x$ and to "flip" a part containing $y$. 


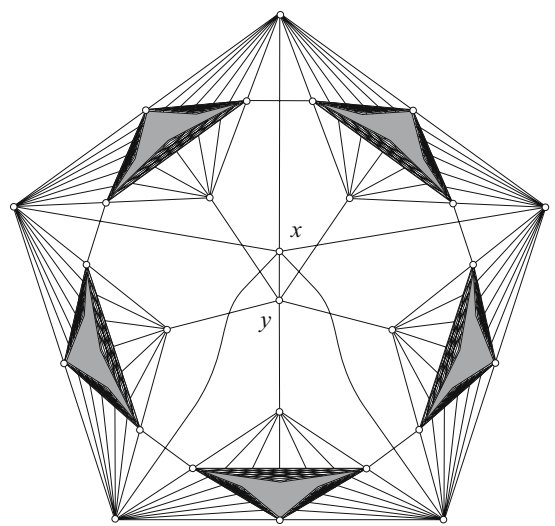

Fig. 1. The graph $Q_{k}$

And maybe some repetition of such changes may be needed. If this would be the only possibility of making the crossing number smaller than the one coming from the planar drawing of $G_{0}$, this would most likely give rise to a polynomial time algorithm for computing the crossing number of near-planar graphs. However, the authors can construct examples, in which additional complications arise.

Despite these examples and despite our NP-hardness result for the weighted case, the following question may still have a positive answer:

Problem 1. Is there a polynomial time algorithm which would determine the crossing number of $G_{0}+x y$ if $G_{0}$ is an unweighted 3-connected planar graph?

\section{Planar Separations and the Dual Distance}

Let $G_{0}$ be a planar graph, $x, y$ distinct vertices of $G_{0}$, and let $Q$ be a subgraph of $G_{0}-x-y$. We say that $Q$ planarly separates vertices $x$ and $y$ if for every embedding of $G_{0}$ in the plane, $x$ and $y$ lie in the interiors of distinct faces of the induced embedding of $Q$.

Let $Q$ be a subgraph of $G$. A $Q$-bridge in $G$ is a subgraph of $G$ that is either an edge not in $Q$ but with both ends in $Q$ (and its ends also belong to the bridge), or a connected component of $G-V(Q)$ together with all edges (and their endvertices in $Q$ ) which have one end in this component and the other end in $Q$. Let $B$ be an $Q$-bridge. Vertices of $B \cap Q$ are vertices of attachment of $B$ (shortly attachments).

Let $C$ be a cycle in $G_{0}-x-y$. Let $B_{x}$ and $B_{y}$ be the $C$-bridges in $G_{0}$ containing $x$ and $y$, respectively. Two $C$-bridges $B$ and $B^{\prime}$ are said to overlap if either (i) $C$ contains four vertices $a, a^{\prime}, b, b^{\prime}$ in this order such that $a$ and $b$ are attachments of $B$ and $a^{\prime}, b^{\prime}$ are attachments of $B^{\prime}$, or (ii) $B$ and $B^{\prime}$ have (at least) three vertices of attachment in common. We define the overlap graph $O\left(G_{0}, C\right)$ of $C$-bridges (see [12]) as the graph whose vertices are the bridges of $C$, and two vertices are adjacent if the two bridges overlap on $C$. Since $G_{0}$ is planar, 
the overlap graph is bipartite. Distinct $C$-bridges are weakly overlapping if they are in the same connected component of $O\left(G_{0}, C\right)$, and in that component they belong to distinct bipartite classes. The following result follows easily from the definitions.

Lemma 1. A cycle $C \subseteq G_{0}-x-y$ planarly separates $x$ and $y$ if and only if $B_{x}$ and $B_{y}$ are weakly overlapping $C$-bridges.

Tutte 17] characterized when $G_{0}+x y$ is non-planar, i.e., when $\operatorname{cr}\left(G_{0}+x y\right) \geq 1$ by proving

Theorem 3 (Tutte [17]). Let $x, y$ be vertices of a planar graph $G_{0}$. Then $G_{0}+x y$ is non-planar if and only if $G_{0}-x-y$ contains a cycle $C$ such that the $C$-bridges of $G$ containing $x$ and $y$, respectively, are overlapping.

Let us observe that $G_{0}+x y$ is non-planar if and only if $G_{0}-x-y$ planarly separates $x$ and $y$. Therefore, the next lemma is closely related to Theorem 3 .

Lemma 2. If $Q \subseteq G_{0}-x-y$ planarly separates $x$ and $y$, then there is a cycle $C \subseteq Q$ that planarly separates $x$ and $y$.

The proof of this lemma is not hard but slightly technical, and we defer it to the full version of this paper.

For a plane graph $G_{0}$, a sequence $Q_{1}, \ldots, Q_{k}$ of edge-disjoint cycles of $G_{0}$ is nested if for $i=1, \ldots, k-1$, all edges of the cycle $Q_{i+1}$ lie in the exterior of $Q_{i}$.

Lemma 3. Suppose that $C$ and $D$ are edge-disjoint cycles that planarly separate vertices $x$ and $y$. Then there exist nested cycles $C_{1}, C_{2} \subseteq C \cup D$ that planarly separate $x$ and $y$.

Again, the proof is deferred for the full version of the paper.

Lemma 4. Let $G_{0}$ be a plane graph. If $Q_{1}, \ldots, Q_{k}$ are edge-disjoint cycles of $G_{0}$ that planarly separate vertices $x$ and $y$ of $G_{0}$, then there are nested edge-disjoint cycles $Q_{1}^{\prime}, \ldots, Q_{k}^{\prime}$ such that $\cup_{i=1}^{k} E\left(Q_{i}^{\prime}\right) \subseteq \cup_{i=1}^{k} E\left(Q_{i}\right)$ and such that $Q_{1}^{\prime}, \ldots, Q_{k}^{\prime}$ planarly separate $x$ and $y$.

Proof. The proof follows rather easily by applying Lemma 3 consecutively on pairs of cycles $Q_{i}, Q_{j}$. One has to make sure that after finitely many steps we get a collection of nested cycles. This is done as follows. First we apply the lemma in such a way that one of the cycles in the family has none of the edges of the other $k-1$ cycles in its interior. After this is done, we repeat the process with the remaining $k-1$ cycles.

After this preparation, we are ready to discuss a dual expression for the dual distance, both for the 3-connected and for the general case.

Theorem 4. Let $G_{0}$ be a planar graph and $x, y \in V(G)$. If $r$ is an integer, then the following statements are equivalent: 
(a) $r \leq d_{0}^{*}(x, y)$.

(b) There exists a family of $r$ edge-disjoint cycles $Q_{1}, \ldots, Q_{r}$ that planarly separate $x$ and $y$.

(c) There exists a family of $r$ nested cycles $Q_{1}, \ldots, Q_{r}$ that planarly separate $x$ and $y$.

Equivalence of (b) and (c) follows from Lemma 4. It is also clear from the definitions (cf. Lemma 1) that (b) implies (a). The proof of the reverse implication that (a) yields (b) is by induction and also gives an efficient algorithm for finding $d_{0}^{*}(x, y)$ nested cycles planarly separating $x$ and $y$. Let us observe that for 3 -connected graphs, the maximum number of nested cycles can be determined by a simple "greedy" process.

Corollary 1. The value of $d_{0}^{*}(x, y)$ is equal to the maximum number of edgedisjoint cycles that planarly separate $x$ and $y$.

The above dual expression for $d_{0}^{*}(x, y)$ is a min-max relation which offers an extension to the weighted case. Suppose that the edges of $G_{0}+x y$ are weighted and that all weights are positive integers. Then we can replace each edge $e \neq x y$ by $w_{e}$ parallel edges (each of weight 1$)$. Let $\tilde{G}_{0}$ be the resulting unweighted graph. It is easy to argue that $d_{0}^{*}\left(G_{0}, x, y\right)$ is equal to $d_{0}^{*}\left(\tilde{G}_{0}, x, y\right) \cdot w_{x y}$. By Corollary [1. this value can be interpreted as the maximum number of edge-disjoint cycles planarly separating $x$ and $y$ in $\tilde{G}_{0}$.

\section{Facial Distance}

In this section we shall prove Theorem 2. First, we need a dual expression for $d^{\prime}(x, y)$ which can be viewed as a surface version of Menger's Theorem.

Proposition 2. Let $G_{0}$ be a plane graph and $x, y \in V\left(G_{0}\right)$ where $y$ lies on the boundary of the exterior face. Let $r$ be the maximum number of vertex-disjoint cycles, $Q_{1}, \ldots, Q_{r}$, contained in $G_{0}-x-y$, such that for $i=1, \ldots, r, x \in \operatorname{int}\left(Q_{i}\right)$ and $y \in \operatorname{ext}\left(Q_{i}\right)$. Then $d^{\prime}(x, y)=r$.

Proof. Since every $(x, y)$-arc intersects every $Q_{i}$, we conclude that $d^{\prime}(x, y) \geq r$. The converse inequality is proved by induction on $d^{\prime}(x, y)$. There is nothing to show if $d^{\prime}(x, y)=0$. Let $F$ be the subgraph of $G_{0}$ containing all vertices and edges that are cofacial with $x$. Then $F$ contains a cycle $Q$ such that $x \in \operatorname{int}(Q)$ and $y \in \operatorname{ext}(Q)$. Delete all vertices and edges of $F$ except $x$, and let $G_{1}$ be the resulting plane graph. It is easy to see that $d_{G_{1}}^{\prime}(x, y)=d_{G_{0}}^{\prime}(x, y)-1$. By the induction hypothesis, $G_{1}$ has $d_{G_{0}}^{\prime}(x, y)-1$ disjoint cycles that contain $x$ in their interior and $y$ in the exterior. By adding $Q$ to this family, we get $d^{\prime}(x, y)$ such cycles. This shows that $d^{\prime}(x, y) \leq r$.

The cycles $Q_{1}, \ldots, Q_{r}$ in Proposition 2 all contain $x$ in their interior and $y$ in their exterior. Therefore, they behave essentially like cycles on a cylinder that 
separate the two boundary components of the cylinder. Hence they are nested cycles separating $x$ and $y$.

The main result of this section, Theorem 2, involves the minimum facial distance taken over all embeddings of $G_{0}$ in the plane. If $G_{0}$ is 3-connected, then $d^{\prime}(x, y)$ is the same for every embedding of $G_{0}$, and Proposition 2 yields a dual expression for the facial distance. For general graphs, we need a similar concept as used in the previous section.

Let $G_{0}$ be a graph and $x, y \in V\left(G_{0}\right)$. Then we define $\rho\left(x, y, G_{0}\right)$ as the largest integer $r$ for which there exists a collection of $r$ vertex-disjoint cycles $Q_{1}, \ldots, Q_{r}$ in $G-x-y$ such that for every $i=1, \ldots, r, x$ and $y$ belong to distinct weakly overlapping bridges of $Q_{i}$. It is convenient to realize that it may be required that the bridges containing $x$ and $y$ indeed overlap (not only weakly overlap), so we get an extension of Tutte's Theorem 3 .

Lemma 5. Let $r=\rho\left(x, y, G_{0}\right)$. Then there exists a collection of $r$ vertexdisjoint cycles $Q_{1}, \ldots, Q_{r}$ in $G_{0}-x-y$ such that for every $i=1, \ldots, r, x$ and $y$ belong to distinct overlapping bridges of $Q_{i}$.

Proof. For $i=1, \ldots, r$, let $B_{x}^{i}$ (resp. $B_{y}^{i}$ ) be the $Q_{i}$-bridge in $G_{0}$ containing $x$ (resp. $y)$. Note that every other cycle $Q_{j}(j \neq i)$ is contained either in $B_{x}^{i}$ or in $B_{y}^{i}$. Therefore we can define a linear order $\prec$ on $\left\{Q_{1}, \ldots, Q_{r}\right\}$ by setting $Q_{i} \prec Q_{j}$ if and only if $Q_{j} \subseteq B_{y}^{i}$. By adjusting indices, we may assume that $Q_{1} \prec Q_{2} \prec \cdots \prec Q_{r}$.

The proof method used in particular by Tutte in [17] is to change each cycle $Q_{i}$ by rerouting it through the $Q_{i}$-bridges distinct from $B_{x}^{i}$ and $B_{y}^{i}$ in such a way that the two bridges with respect to the new cycle still weakly overlap, but contain more vertices. The actual goal is to minimize the number $t$ of edges that are neither on the cycle nor in one of these two bridges. If $B_{x}^{i}$ and $B_{y}^{i}$ do not overlap but are weakly overlapping, it is possible to decrease $t$. It follows that after a series of changes, that do not affect any of the other cycles, the "big" bridges $B_{x}^{i}$ and $B_{y}^{i}$ overlap. We refer to [7] and to [1] for an algorithmic treatment showing that these changes can be made in linear time.

The following lemma, whose proof is deferred to the full paper, is the analogue of Theorem 4 .

Lemma 6. $d_{0}^{\prime}(x, y)=\rho\left(x, y, G_{0}\right)$.

We are ready for the proof of Theorem 2 .

Proof. (of Theorem 2). It has been shown before that $\operatorname{cr}\left(G_{0}+x y\right) \leq d_{0}^{*}(x, y)$. The heart of the proof is to show that $d_{0}^{\prime}(x, y)$ is a lower bound on $\operatorname{cr}\left(G_{0}+x y\right)$. Let $r=d_{0}^{\prime}(x, y)$. Lemmas 5 and 6 show that there are $r$ vertex-disjoint $Q_{1}, \ldots, Q_{r}$ such that for every $i=1, \ldots, r, x$ and $y$ belong to distinct overlapping bridges of $Q_{i}$. Let us denote these overlapping $Q_{i}$-bridges $B_{x}^{i}$ and $B_{y}^{i}$ as we did above. To simplify the notation in the sequel, we define $Q_{0}=\{x\}$ and $Q_{r+1}=\{y\}$. Since $B_{x}^{i}$ and $B_{y}^{i}$ overlap, one of the following cases occurs: 
(i) There are paths $P_{1}^{+}, P_{2}^{+} \subseteq B_{y}^{i}$ joining $Q_{i}$ with $Q_{i+1}$, and there are paths $P_{1}^{-}, P_{2}^{-} \subseteq B_{x}^{i}$ joining $Q_{i}$ with $Q_{i-1}$ such that the ends of these pairs of paths on $Q_{i}$ interlace.

(ii) When the bridges $B_{x}^{i}$ and $B_{y}^{i}$ have precisely three vertices of attachment, they may overlap only because their attachments $a, b, c$ on $Q_{i}$ coincide. In that case, we have paths $P_{1}^{+}, P_{2}^{+}, P_{3}^{+}$in $B_{y}^{i}$ (resp. paths $P_{1}^{-}, P_{2}^{-}, P_{3}^{-}$in $B_{x}^{i}$ ) joining $a, b, c$ with $Q_{i+1}$ (resp. $\left.Q_{i-1}\right)$.

If Case (i) occurs, let $S^{i}$ be the union of the paths $P_{1}^{-}$and $P_{2}^{-}$and let $R^{i}$ be the union of the paths $P_{1}^{+}$and $P_{2}^{+}$. If Case (ii) occurs, we define $S^{i}$ and $R^{i}$ similarly, as the union of the three paths in (ii) certifying the overlapping.

Suppose that we have a clean drawing of $G_{0}+x y$ in the plane. If two cycles $Q_{i}$ and $Q_{i+1}$ intersect, then they make at least two crossings, and we declare one of them to be a crossing of type $i$, and the other one a crossing of type $i+1$. If two edges of the same cycle $Q_{i}$ cross, we declare that crossing to be of type $i$ as well. If an edge $e \notin E\left(Q_{1} \cup \cdots \cup Q_{r}\right) \cup R^{i} \cup S^{i} \cup R^{i-1} \cup S^{i+1}$ (including the possibility that $e=x y$ ) crosses an edge of $Q_{i}$, we also declare the crossing to be of type $i$. Finally, if two edges, $e \in E\left(Q_{i-1} \cup S^{i}\right)$ and $f \in E\left(Q_{i+1} \cup R^{i}\right)$ cross, we also say that the crossing is of type $i$. Observe that by this definition, none of the crossings is of two different types (but for some of the crossings, the type may not have been specified).

Our goal is to show that for every $i=1, \ldots, r$, there is a crossing of type $i$. This will show that there are at least $r$ crossings, so the theorem holds.

Suppose, reductio ad absurdum, that there is no crossing of type $i(1 \leq i \leq r)$. Then $Q_{i}$ does not cross itself and both $x$ and $y$ are in the interior of $Q_{i}$ (say) since the edge $x y$ does not cross $Q_{i}$. Moreover, $Q_{i}$ is not crossed by any of the other cycles $Q_{j}$. Suppose now that $Q_{i-1}$ and $Q_{i+1}$ are both inside $Q_{i}$ (or both outside). Then it is easy to see that a crossing of type $i$ occurs between an edge $e \in E\left(Q_{i-1} \cup S^{i}\right)$ and an edge $f \in E\left(Q_{i+1} \cup R^{i}\right)$. This shows that one of $Q_{i-1}$ and $Q_{i+1}$ is inside, while the other one is outside $Q_{i}$. We may assume that $Q_{i+1}$ is inside and $Q_{i-1}$ is outside $Q_{i}$. There is a path from $Q_{i-1}$ to $x$ that is disjoint from $V\left(Q_{i}\right)$ and does not use edges in $S^{i}$ or in $R^{i-1}$. This path must clearly cross $Q_{i}$, and yields a crossing of type $i$. This contradiction completes the proof.

As a corollary we get a generalization of Riskin's Theorem 1 .

Corollary 2. If the graph $G_{0}-x-y$ has maximum degree 3 , then $\operatorname{cr}\left(G_{0}+x y\right)=$ $d_{0}^{\prime}(x, y)=d_{0}^{*}(x, y)$. In particular, the crossing number of $G_{0}+x y$ is computable in linear time.

Another corollary is an approximation formula for the crossing number of nearplanar graphs if the maximum degree is bounded.

Corollary 3. If the graph $G_{0}-x-y$ has maximum degree $\Delta$, then $d_{0}^{\prime}(x, y) \leq$ $\operatorname{cr}\left(G_{0}+x y\right) \leq \frac{\Delta}{2} d_{0}^{\prime}(x, y)$.

Proof. Observe that $d_{0}^{*}(x, y) \leq \frac{\Delta}{2} d_{0}^{\prime}(x, y)$ because there are at most $\frac{\Delta}{2}$ edgedisjoint cycles through any vertex and $d_{0}^{*}(x, y)$ is defined by a collection of $d_{0}^{*}(x, y)$ nested cycles (c.f. Theorem 4). 
Corollary 3 is an improvement of a theorem of Hliněný and Salazar [6] who proved analogous result with the factor $\Delta$ instead of $\Delta / 2$.

A graph $G$ is said to be $d$-apex if $G$ has a vertex $v$ of degree at most $d$ such that $G-v$ is planar. Let us observe that every near-planar graph is essentially 2-apex (subdivide the "non-planar" edge).

Problem 2. Is there a result similar to Corollary 2 for 3-apex cubic graphs?

\section{The Minor Crossing Number and $d^{\prime}$}

Structural graph theory based on the Robertson and Seymour theory of graph minors gives powerful results in relation to topological realizations of graphs. However, it does not work well with crossing numbers. To overcome this deficiency, Bokal et al. 2] introduced a related notion of the minor crossing number, $\operatorname{mcr}(G)$, which is defined as the minimum of $\operatorname{cr}(H)$ taken over all graphs $H$ that contain $G$ as a minor.

It is easy to see that $\operatorname{mcr}\left(G_{0}+x y\right) \leq d_{0}^{\prime}(x, y)$. However, a proof along similar lines as the proof of Theorem 2 shows even more intimate relationship.

Theorem 5. $\operatorname{mcr}\left(G_{0}+x y\right)=d_{0}^{\prime}(x, y)$.

\section{NP-Hardness of wcr $(\cdot)$ for Near-Planar Graphs}

Consider the following decision problem:

Weighted Crossing Number

Input: $G, k$, where $G$ is an edge-weighted graph and $k>0$.

Question: Is $\operatorname{wcr}(G) \leq k$ ?

This problem is NP-complete because it generalizes the problem Crossing Number, which is NP-complete [3. We will see that this problem remains NP-complete when restricted to near-planar graphs. We will use the notation $[n]=\{1, \ldots, n\}$.

Let $a_{1}, \ldots, a_{n}$ be natural numbers, and let $S=\sum_{i \in[n]} a_{i}$. We define the edgeweighted graph $G\left(a_{1}, \ldots, a_{n}\right)$ as follows (Fig. 2):

- its vertices are $u_{1}, \ldots, u_{n}$ and $v_{1}, \ldots, v_{n}$;

- there is a Hamiltonian cycle $Q=u_{1} u_{2} \cdots u_{n} v_{1} v_{2} \cdots v_{n} u_{1}$, each edge with weight $S^{2}$;

- there are edges $e_{i}=u_{i} v_{i}$ with weight $a_{i}$ for each $i \in[n]$;

It is easy to note that $G\left(a_{1}, \ldots, a_{n}\right)-u_{1} v_{n}$ planar, and hence $G\left(a_{1}, \ldots, a_{n}\right)$ is near-planar. For any subset of indices $I \subseteq[n]$, let $\sigma_{I}:=\sum_{i \in I} a_{i}$. Consider a clean drawing $\mathcal{D}_{0}$ of $G$ such that $\operatorname{wcr}(G)=\operatorname{wcr}\left(\mathcal{D}_{0}\right)$. It is easy to see that no edge of $Q$ participates in a crossing, and therefore each edge $e_{i}$ is contained either in the interior or in the exterior of the simple closed curve defined by $Q$. Using that all the edges in the interior (or the exterior) of $Q$ must cross each other, we can show the following property. 


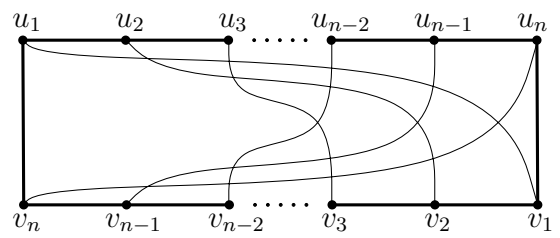

Fig. 2. The graph $G\left(a_{1}, \ldots, a_{n}\right)$ with the cycle $Q$ bolder

Lemma 7. It holds that

$$
2 \cdot \operatorname{wcr}\left(G\left(a_{1}, \ldots, a_{n}\right)\right)=\min _{I \subseteq[n]}\left\{\left(\sigma_{I}\right)^{2}+\left(\sigma_{[n] \backslash I}\right)^{2}\right\}-\sum_{i \in[n]} a_{i}^{2} .
$$

Lemma 8. It holds

$$
\mathrm{wcr}\left(G\left(a_{1}, \ldots, a_{n}\right)\right)=S^{2} / 4-\sum_{i \in[n]} a_{i}^{2} / 2
$$

if and only if there exists $I \subset[n]$ such that $\sigma_{I}=\sigma_{[n] \backslash I}=S / 2$.

Proof. Note that

$\min _{I \subseteq[n]}\left\{\left(\sigma_{I}\right)^{2}+\left(\sigma_{[n] \backslash I}\right)^{2}\right\} \geq \min \left\{A^{2}+B^{2} \mid A+B=S, A \geq 0, B \geq 0\right\}=S^{2} / 2$,

and there is equality if and only if there is some $I \subset[n]$ such that $\sigma_{I}=\sigma_{[n] \backslash I}=$ $S / 2$. The result then follows from Lemma 7 .

Theorem 6. The problem Weighted Crossing Number is NP-complete for near-planar graphs.

Proof. A standard planarizing argument shows that the problem Weighted Crossing Number is in NP. To show NP-hardness, consider the following NPcomplete problem [4.

\section{PARTITION}

Input: natural numbers $a_{1}, \ldots, a_{n}$.

Question: is there $I \subset[n]$ such that $\sum_{i \in I} a_{i}=\sum_{i \in[n] \backslash I} a_{i}$ ?

Consider the function $\phi$ that maps the input $a_{1}, \ldots, a_{n}$ for PARTition into the input

$$
G\left(a_{1}, \ldots, a_{n}\right), S^{2} / 4-\sum_{i \in[n]} a_{i}^{2} / 2
$$

for Weighted Crossing Number . Clearly, $\phi$ can be computed in polynomial time. Because of Lemma 8 both problems have the same answer. Therefore we have a polynomial time reduction from Partition to Weighted Crossing NumBer that only uses near-planar graphs. 


\section{References}

1. Bhatt, S.N., Leighton, F.T.: A framework for solving VLSI graph layout problems. J. Comput. System Sci. 28(2), 300-343 (1984)

2. Bokal, D., Fijavž, G., Mohar, B.: The minor crossing number. SIAM J. Discret. Math. 20(2), 344-356 (2006)

3. Garey, M.R., Johnson, D.S.: Crossing number is NP-complete. SIAM J. Alg. Discr. Meth. 4, 312-316 (1983)

4. Garey, M.R., Johnson, D.S.: Computers and Intractability: A Guide to the Theory of NP-Completeness. W. H. Freeman \& Co., New York (1979)

5. Gutwenger, C., Mutzel, P., Weiskircher, R.: Inserting an edge into a planar graph. Algorithmica 41, 289-308 (2005)

6. Hliněný, P., Salazar, G.: On the crossing number of almost planar graphs. In: Kaufmann, M., Wagner, D. (eds.) GD 2006. LNCS, vol. 4372, pp. 162-173. Springer, Heidelberg (2007)

7. Juvan, M., Marinček, J., Mohar, B.: Elimination of local bridges. Math. Slovaca 47, 85-92 (1997)

8. Leighton, F.T.: Complexity issues in VLSI. MIT Press, MA (1983)

9. Leighton, F.T.: New lower bound techniques for vlsi. Math. Systems Theory 17, 47-70 (1984)

10. Liebers, A.: Planarizing graphs - a survey and annotated bibliography. J. Graph Algorithms Appl. 5, 74pp. (2001)

11. Mishra, B., Tarjan, R.E.: A linear-time algorithm for finding an ambitus. Algorithmica 7(5\&6), 521-554 (1992)

12. Mohar, B., Thomassen, C.: Graphs on Surfaces. Johns Hopkins University Press, Baltimore (2001)

13. Mohar, B.: On the crossing number of almost planar graphs. Informatica 30, 301303 (2006)

14. Riskin, A.: The crossing number of a cubic plane polyhedral map plus an edge. Studia Sci. Math. Hungar. 31, 405-413 (1996)

15. Shahrokhi, F., Sýkora, O., Székely, L.A., Vrt'o, I.: Crossing numbers: bounds and applications. In: Barany, I., Böröczky, K. (eds.) Intuitive geometry (Budapest, 1995). Bolyai Society Mathematical Studies, vol. 6, pp. 179-206. Akademia Kiado (1997)

16. Székely, L.A.: A successful concept for measuring non-planarity of graphs: The crossing number. Discrete Math. 276, 331-352 (2004)

17. Tutte, W.T.: Separation of vertices by a circuit. Discrete Math. 12, 173-184 (1975)

18. Vrt'o, I.: Crossing number of graphs: A bibliography, ftp://ftp.ifi.savba.sk/pub/imrich/crobib.pdf 